\title{
Indications for sublobar resection of clinical stage IA radiologic pure-solid lung adenocarcinoma
}

\author{
Aritoshi Hattori, MD, Takeshi Matsunaga, MD, Kazuya Takamochi, MD, Shiaki Oh, MD, and \\ Kenji Suzuki, MD
}

\begin{abstract}
Objectives: The aim of this study was to identify clinical factors associated with lepidic growth in resected clinical stage IA radiologic pure-solid lung adenocarcinoma for identifying a possible sublobar resection candidate in the population.

Methods: Clinicopathologic data were reviewed for 200 surgically resected clinical stage IA pure-solid lung adenocarcinomas. Radiologic pure-solid tumor was defined as a tumor without a ground-glass opacity component, that is, a consolidation tumor ratio equal to 1.0. Lepidic predominant adenocarcinoma included adenocarcinomas in situ, minimally invasive adenocarcinomas, and lepidic predominant invasive adenocarcinomas.
\end{abstract}

Results: A total of 57 patients $(29 \%)$ had lepidic predominant adenocarcinoma. The 5-year overall survival of clinical stage IA pure-solid adenocarcinoma was $83.4 \%$ and that of lepidic predominant adenocarcinoma and nonlepidic predominant adenocarcinoma was $98.1 \%$ versus $76.6 \%(P=.0012)$. A multivariate analysis revealed that maximum standardized uptake value was an independently significant variable of lepidic predominant adenocarcinoma $(P<.0001)$ and a significant prognostic factor $(P=.034)$. The predictive criterion of lepidic predominant adenocarcinoma was maximum standardized uptake value 3.3 or less based on a receiver operating characteristic curve, and 77 patients $(39 \%)$ who met this criterion showed less pathologic invasiveness regarding lymphatic $(P=.0012)$ and vascular $(P<.0001)$ invasions, nodal metastasis $(P=.0007)$, and better overall survival than those who did not (maximum standardized uptake value $\leq 3.3$ vs $>3.3$ rates being $91.7 \%$ vs $78.6 \%, P=.0031$ ). Moreover, the 3 -year locoregional recurrence-free survival of the sublobar resection arm was significantly worse than that of the lobectomy arm when the tumor showed maximum standardized uptake value greater than $3.3(62.7 \%$ vs $82.9 \%, P=.0281)$.

Conclusions: Higher maximum standardized uptake value may be useful for identifying patients with clinical stage IA radiologic pure-solid lung adenocarcinoma in whom sublobar resection should not be considered, even if technically feasible. (J Thorac Cardiovasc Surg 2017;154:1100-8)

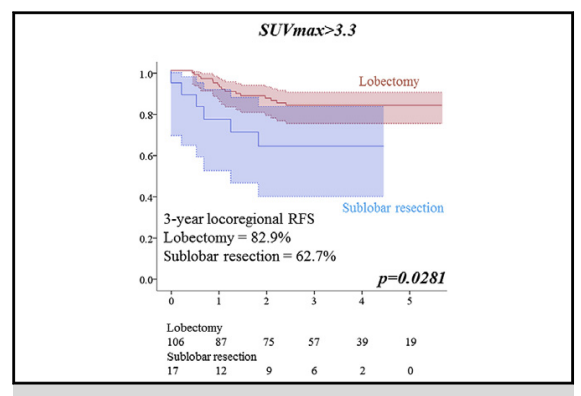

Lobectomy showed better local control in patients with high standardized uptake value.

\section{Central Message}

Higher SUVmax may be useful for identifying patients with stage IA radiologic pure-solid lung adenocarcinoma in whom sublobar resection should not be considered.

\section{Perspective}

The primary aim of this study was to elucidate clinical factors associated with lepidic growth in resected clinical stage IA radiologic puresolid lung adenocarcinoma. As a result, SUVmax was a promising clinical variable for identifying lepidic predominance among them and was associated with differential survival in patients with sublobar versus lobar resection.

See Editorial Commentary page 1109.

See Editorial page 1075 .
From the Department of General Thoracic Surgery, Juntendo University School of Medicine, Tokyo, Japan.

This work was supported in part by a Grant-in-Aid for Cancer Research from the Ministry of Health, Labour and Welfare, Japan, the Smoking Research Foundation, and the National Cancer Center Research and Development Fund (26-A-4).

Read at the 96th Annual Meeting of The American Association for Thoracic Surgery, Baltimore, Maryland, May 14-18, 2016.

Received for publication June 16, 2016; revisions received Feb 12, 2017; accepted for publication March 12, 2017; available ahead of print June 16, 2017.

Address for reprints: Kenji Suzuki, MD, Department of General Thoracic Surgery, Juntendo University School of Medicine, 1-3, Hongo 3-chome, Bunkyo-ku, Tokyo, 113-8431, Japan (E-mail: kjsuzuki@juntendo.ac.jp). 0022-5223/\$36.00

Copyright @ 2017 Published by Elsevier Inc. on behalf of The American Association for Thoracic Surgery

http://dx.doi.org/10.1016/j.jtcvs.2017.03.153
Lung adenocarcinomas have recently become the most common histologic subtypes of non-small cell lung cancers. ${ }^{1,2}$ Since 2011, the International Association for the Study of Lung Cancer, American Thoracic Society, and European Respiratory Society (IASLC/ATS/ETS) have characterized

Scanning this QR code will take you to supplemental videos. 


$\begin{array}{ll}\text { Abbreviations and Acronyms } \\ \text { AIS } & =\text { adenocarcinoma in situ } \\ \text { CT } & =\text { computed tomography } \\ \text { FDG } & 18 \text { F-fluorodeoxyglucose } \\ \text { GGO } & \text { ground-grass opacity } \\ \text { IASLC/ATS/ETS = } & \text { International Association for } \\ & \text { the Study of Lung Cancer, } \\ & \text { American Thoracic Society, } \\ & \text { and European Respiratory } \\ & \text { Society } \\ & =\text { Japan Clinical Oncology Group } \\ = & \text { lepidic predominant } \\ \text { JCOG } & \text { adenocarcinoma } \\ \text { LPA } & \text { minimally invasive } \\ & \text { adenocarcinoma } \\ \text { MIA } & =\text { overall survival } \\ \text { OS } & =\text { positron emission tomography } \\ \text { PET } & =\text { recurrence-free survival } \\ \text { RFS } & \text { maximum standardized uptake } \\ \text { SUVmax } & \text { value }\end{array}$

lung adenocarcinomas as a heterogeneous group on the basis of the comprehensive histologic subtypes. ${ }^{2}$ This classification associates well with the prognosis and indicates a favorable oncologic outcome for adenocarcinoma in situ (AIS), minimally invasive adenocarcinoma (MIA), and lepidic predominant invasive adenocarcinoma.

Meanwhile, the presence of a ground-grass opacity (GGO) component is considered to denote a good prognosis, and in most cases the pathologic features are minimally invasive because of a significant correlation with histologic lepidic growths. $^{3-7}$ They are considered to be feasible candidates of sublobar resection. ${ }^{8}$ Traditionally, however, a radiologic pure-solid lung adenocarcinoma without any GGO component is assumed to show a highly malignant nature, and postoperative nodal involvement is found in approximately $15 \%$ to $20 \%$ of cases, even in those with small-sized lesions. ${ }^{4,9-11}$

Several institutional retrospective studies have recently suggested that survival and recurrence may be identical between lobectomy and sublobar resection in patients with small-sized lung cancers. ${ }^{12-15}$ To elucidate the efficacy of sublobar resection for small-sized lung cancers based on these retrospective studies, prospective clinical trials have been conducted for c-T1a radiologic invasive part-solid or pure-solid lung cancers in both Japan ${ }^{16}$ and the United States. ${ }^{17}$ Unlike a part-solid lung cancer, however, whether sublobar resection is an appropriate surgical mode for radiologic pure-solid lung cancers remains unclear because of their highly invasive nature. ${ }^{10} \mathrm{~A}$ major problem in routine practice is the fact that identifying histologic lepidic predominance in radiologic pure-solid lung adenocarcinoma on the basis of preoperative findings on thin-section computed tomography (CT) scan is a challenge. This is due to a lack of useful clinical parameters other than GGO components. If the event could be identified preoperatively, that would enable surgeons to select appropriate candidates of sublobar resections even among patients with radiologic pure-solid lung adenocarcinomas.

Therefore, in the current study, we retrospectively reviewed surgically resected clinical stage IA radiologic pure-solid lung adenocarcinoma at Juntendo University School of Medicine. The primary aim of this study was to identify clinical factors associated with histologic lepidic growth in resected, clinical stage IA radiologic pure-solid lung adenocarcinoma. The secondary aim was to determine whether these clinical factors for histologic lepidic growth were associated with differential survival in the overall population and in patients who underwent sublobar versus lobar resection.

\section{MATERIALS AND METHODS Study Population}

Between January 2008 and March 2013, 238 patients underwent surgical resection for clinical stage IA radiologic pure-solid lung adenocarcinomas in a single institute. Among them, 200 patients with a preoperative evaluation of 18F-fluorodeoxyglucose positron emission tomography (FDG-PET) were retrospectively evaluated in this study (Figure 1). Any clinical stage IA lung adenocarcinoma with a GGO component was excluded from the study. Tumors without preoperative histologic confirmation were all proven by intraoperative frozen-section diagnosis. The database has been maintained prospectively, and missing data were missing for the variables in this study. The inclusion criteria were preoperative staging determined by thin-section CT and FDG-PET/CT scans, and complete resection without preoperative chemotherapy or radiotherapy. With regard to the clinical nodal assessment, clinical N0 meant nonenlarged lymph nodes on CT scan and negative FDG uptake by the lymph nodes. Invasive modalities for mediastinal lymph node staging, such as mediastinoscopy or endobronchial ultrasound-guided transbronchial needle aspiration, were not routinely used preoperatively because they were evaluated by thin-section CT or FDG-PET/CT. The medical record of each patient was reviewed retrospectively under a waiver of authorization approved by the institutional review board of Juntendo University School of Medicine, Tokyo, Japan.

\section{Radiologic Evaluations on Thin-Section Computed Tomography Scan}

For all patients, findings of the preoperative thin-section CT scan were reviewed in detail by the authors (A.H., T.M., and K.S.). Tumor size was determined preoperatively on the basis of the thin-section CT scan with 2-mm collimation at Juntendo University School of Medicine. The lung was photographed with a window level of -500 to $-700 \mathrm{H}$ and a window depth of 1000 to $2000 \mathrm{H}$ as a "lung window," and a window level of 30 to $60 \mathrm{H}$ and a window depth of 350 to $600 \mathrm{H}$ as a "mediastinal window." The consolidation tumor ratio was defined as the ratio of the maximum size of consolidation to the maximum tumor size on thin-section CT scan. ${ }^{7}$ In the current study, a radiologic pure-solid tumor was defined as a lung tumor showing only consolidation without GGO on thin-section CT, that is, consolidation tumor ratio was equal to $1.0 .^{4,9,10}$

\section{Positron Emission Tomography}

Positron emission tomography (PET) was performed, and the maximum standardized uptake value (SUVmax) was recorded for all patients in this 


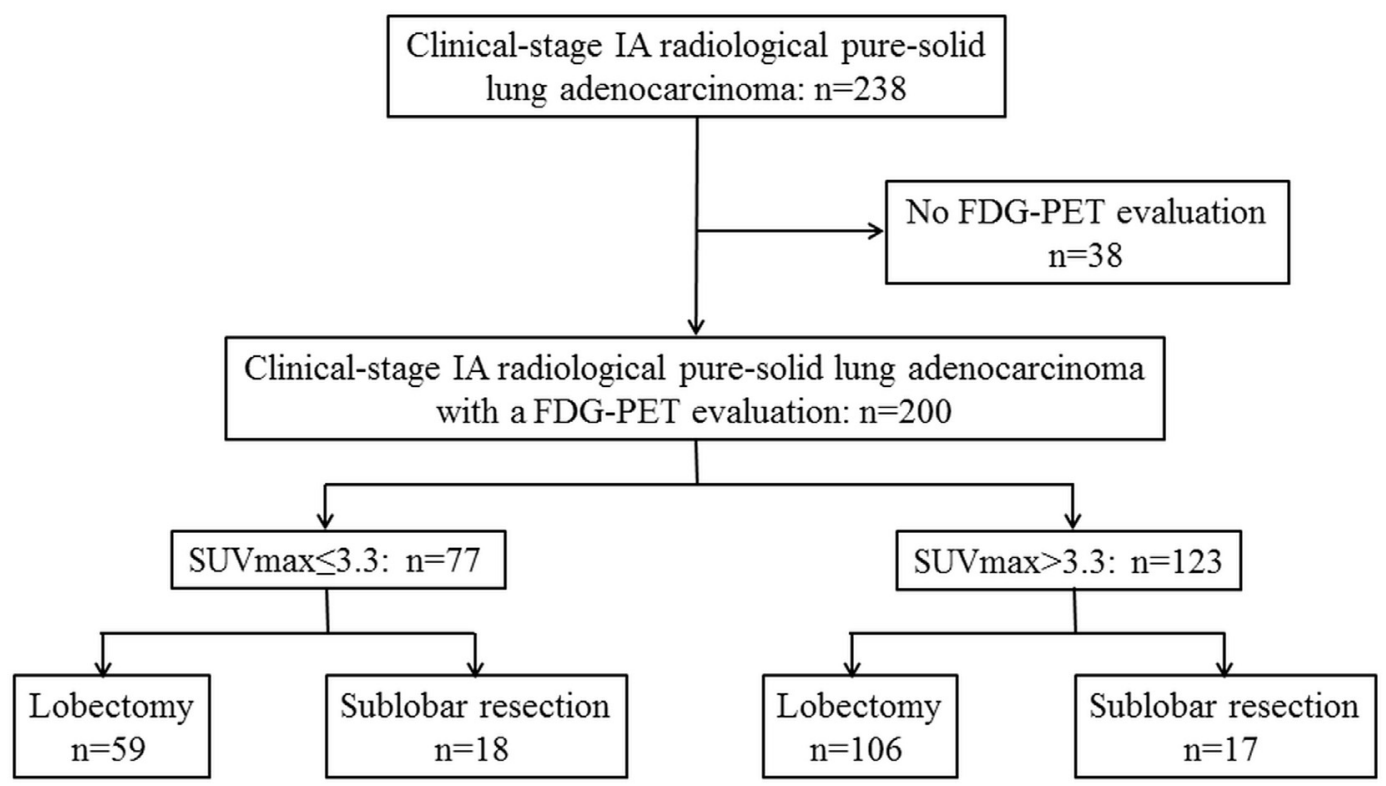

FIGURE 1. Flow chart of patients in the study. FDG-PET, 18F-Fluorodeoxyglucose positron emission tomography; SUVmax, maximum standardized uptake value.

study. In all cases, a PET/CT scan was performed at the Yotsuya Medical Cube (Tokyo, Japan) to remove the standardized uptake value variability among interinstitutional PET scanners. The technique used for the FDG-PET/CT scanning was as follows: All patients fasted for at least 6 hours before the FDG injection to minimize their blood insulin levels and normal tissue glucose uptake. The subjects were injected intravenously with $3.5 \mathrm{MBq} / \mathrm{kg}$ of ${ }^{18} \mathrm{~F}-\mathrm{FDG}$, and static emission images were obtained 60 minutes after the injection. Image acquisition was then performed using a Discovery ST PET/CT scanner (GE Medical Systems, Waukesha, Wis). Emission scanning was performed from the head to mid-thigh in 6 bed positions. The acquired PET data were reconstructed to volumetric images with a two-dimensional-OSEM (ordered-subsets expectationmaximization) algorithm (2 iterations/15 subsets) incorporating a CT-based attenuation correction. All PET/CT images were interpreted by 1 or 2 experienced nuclear medicine radiologists. A workstation (Xeleris; Elegems, Haifa, Israel) was used for image display and analysis, and the SUVmax of the primary tumor was obtained.

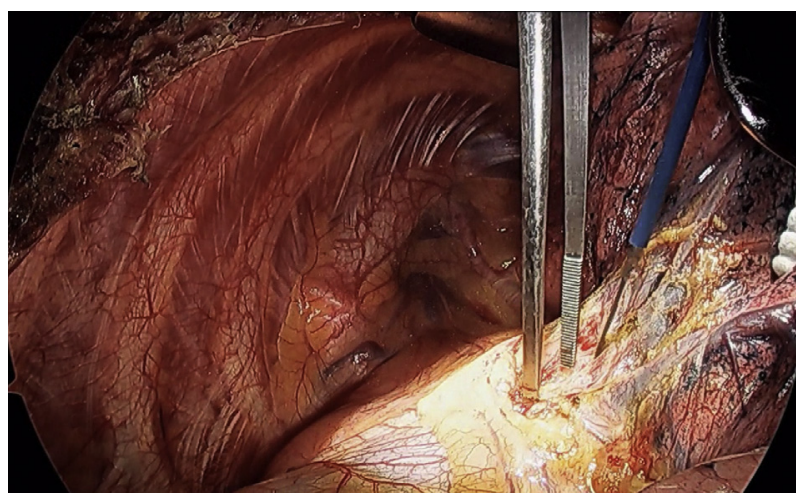

VIDEO 1. The video of left upper division segmentectomy for lung cancer. Video available at: http://www.jtcvsonline.org/article/S0022-5223(17) 30899-1/addons.

\section{Operation Policy}

Lobectomy with systemic lymph node dissection is a standard surgical mode for a radiologically pure-solid tumor in patients at Juntendo University School of Medicine, whereas segmentectomy is now performed for part-solid or pure-solid lung cancers according to the Japan Clinical Oncology Group (JCOG 0802 ${ }^{16}$ ) (Videos 1 and 2). Furthermore, sublobar resection is selected as an optimal treatment strategy for peripheral pure-solid lesions if the tumor is located favorably for complete resection with a sublobar approach and is intraoperatively assessed as N0 by frozen-section evaluation. In contrast, it is occasionally indicated for some patients with pure-solid tumor who are not lobectomy tolerable, such as very elderly patients and high-risk patients with cardiopulmonary disease.

\section{Pathologic Evaluations}

All patients in the present study were reclassified according to the TNM Classification of Malignant Tumors, 7th edition. ${ }^{18}$ Clinical stage IA pure-solid lung adenocarcinomas were classified according to the IASLC/ ATS/ETS classification as AIS and MIA; invasive adenocarcinomas were

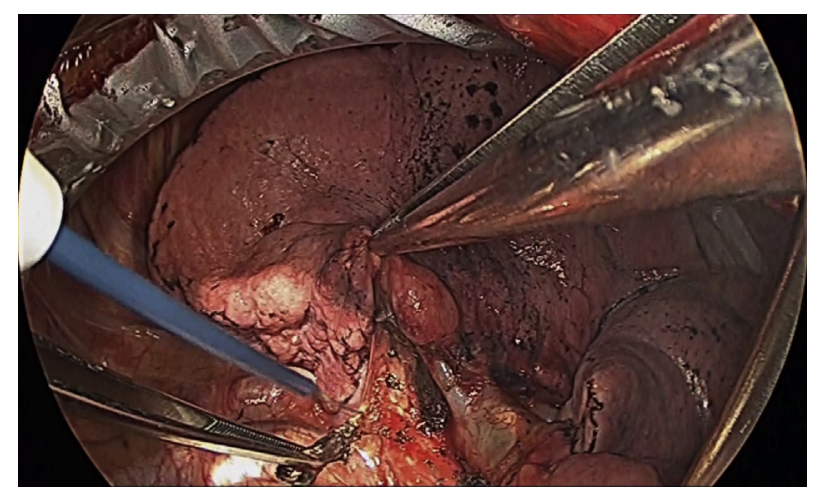

VIDEO 2. The video of right upper lobectomy for lung cancer. Video available at: http://www.jtcvsonline.org/article/S0022-5223(17)30899-1/addons. 
subdivided into lepidic predominant and other subtypes. ${ }^{2}$ Invasive adenocarcinomas were classified according to the predominant subtype after comprehensive histologic subtyping, which implies a semiquantitative estimation of the percentage of different subtypes in $5 \%$ increments. ${ }^{2}$ In the current study, we defined lepidic predominant adenocarcinoma (LPA) as a tumor that showed the lepidic component most frequently including AIS, MIA and lepidic predominant invasive adenocarcinoma; non-LPA was defined as other invasive adenocarcinoma.

\section{Follow-up Policy}

The routine follow-up evaluation included a physical examination, chest radiography, chest CT scan, and blood tests including measurements of tumor markers every 6 to 12 months. If any symptom or sign of recurrence was observed, further evaluation was performed, including CT, brain magnetic resonance imaging, and PET. Locoregional recurrence was defined as occurrence within the residual same lobe and hilum or mediastinal lymph nodes, which was essentially diagnosed by cytologic or histologic confirmation based on biopsy or surgical resection.

\section{Statistics}

Chi-square test was used to compare the factors. Unpaired $t$ test was used if continuous variables were normally distributed, and if not, Wilcoxon rank-sum test was used. Multivariable logistic regression model was fit to identify the clinical factors of LPA using SPSS Statistics 21 (IBM Inc, New York, NY). Forward and backward stepwise procedures were used to determine the combination of factors that were essential for identifying LPA and the survival outcomes. Overall survival (OS) and recurrence-free survival (RFS) functions were estimated using the Kaplan-Meier method and compared by log-rank test across the different groups. The date of surgical resection was set as the starting point and the date of death or survival follow-up as the end point of OS. Moreover, the time of occurrence of the first event including relapse and death, or

TABLE 1. Comparison of clinicopathologic factors based on the lepidic component frequency

\begin{tabular}{|c|c|c|c|}
\hline \multirow[b]{2}{*}{ Factors } & \multicolumn{2}{|c|}{ c-Stage IA pure-solid lung adenocarcinoma } & \multirow[b]{2}{*}{$P$ value } \\
\hline & LPA $(\mathbf{N}=\mathbf{5 7})$ & Non-LPA $(N=143)$ & \\
\hline \multicolumn{4}{|l|}{ Clinical variables } \\
\hline Age (y) & $66.1 \pm 9.9(44-88)$ & $67.2 \pm 10.1(35-89)$ & .522 \\
\hline Sex (male) & $27(47)$ & $92(64)$ & .038 \\
\hline Side (right) & $36(63)$ & $81(57)$ & .430 \\
\hline Location (peripheral/central) & $46(81) / 11(19)$ & $93(65) / 50(35)$ & .040 \\
\hline \multicolumn{4}{|l|}{ Lobe } \\
\hline RU/RM/RL & $18(32) / 4(7) / 14(24)$ & $46(32) / 10(7) / 25(17)$ & .801 \\
\hline LU/LL & $13(23) / 8(14)$ & $35(25) / 27(19)$ & \\
\hline Pack-y of smoking & $0 \pm 30.0(0-95)$ & $30.0 \pm 50.0(0-180)$ & $<.001$ \\
\hline COPD (stage I) & $49(86)$ & $107(75)$ & .093 \\
\hline CEA (ng/mL) & $2.3 \pm 2.6(0.6-14.3)$ & $3.8 \pm 6.5(0.7-82.7)$ & $<.001$ \\
\hline SUVmax & $2.5 \pm 3.2(0.7-18.5)$ & $5.6 \pm 5.6(0.7-23.4)$ & $<.001$ \\
\hline Maximum tumor size (mm) & $17.5 \pm 6.0(9-30)$ & $20.5 \pm 6.0(7-30)$ & .002 \\
\hline Clinical T status (c-T1a/c-T1b) & $40(70) / 17(30)$ & $75(52) / 68(48)$ & .063 \\
\hline Radiologic pleural tail (present) & $25(44)$ & $77(54)$ & .214 \\
\hline Air bronchogram (absent) & $42(74)$ & $73(58)$ & .052 \\
\hline History of cancer (present) & $4(7)$ & $23(16)$ & .110 \\
\hline \multicolumn{4}{|l|}{ Operative variable } \\
\hline Lobectomy/sublobar resection & $42(74) / 15(26)$ & $123(86) / 30(14)$ & .062 \\
\hline \multicolumn{4}{|l|}{ Pathologic variables } \\
\hline \multicolumn{4}{|l|}{ Histologic classification } \\
\hline AIS & $3(5)$ & 0 & $<.001$ \\
\hline MIA & $3(5)$ & 0 & \\
\hline Lepidic predominant invasive ad & $51(90)$ & 0 & \\
\hline Acinar predominant & 0 & $60(42)$ & \\
\hline Papillary predominant & 0 & $43(30)$ & \\
\hline Solid predominant & 0 & $40(28)$ & \\
\hline Nodal involvement (N1/N2) & $1(2) / 4(7)$ & $19(13) / 26(18)$ & .003 \\
\hline Lymphatic invasion (present) & $17(30)$ & $69(48)$ & .018 \\
\hline Vascular invasion (present) & $13(23)$ & $81(57)$ & $<.001$ \\
\hline \multicolumn{4}{|l|}{ Pathologic stage } \\
\hline IA/IB & $46(81) / 5(9)$ & $62(43) / 29(20)$ & $<.001$ \\
\hline IIA/IIB & $1(2) / 0(0)$ & $18(13) / 6(4)$ & \\
\hline IIIA/IIIB & $4(6) / 1(2)$ & $27(19) / 1(1)$ & \\
\hline
\end{tabular}

Categoric data are shown as numbers $(\%)$, and continuous data are shown as mean \pm standard deviation if normally distributed and median \pm interquartile range if not normally distributed (range). The following factors were analyzed as a continuous variable: age, pack-y of smoking, CEA, SUVmax, and maximum tumor size. LPA, Lepidic predominant adenocarcinoma; $R U$, right upper; $R M$, right middle; $R L$, right lower; $L U$, left upper; $L L$, left lower; $C O P D$, chronic obstructive pulmonary disease; $C E A$, carcinoembryonic antigen; SUVmax, maximum standardized uptake value; AIS, adenocarcinoma in situ; MIA, minimally invasive adenocarcinoma. $* P$ value in chi-square test, Student $t$ test, or Wilcoxon rank-sum test. 
TABLE 2. Univariable and multivariable analyses for the prediction of lepidic predominant clinical stage IA pure-solid lung adenocarcinoma

\begin{tabular}{|c|c|c|c|c|c|c|}
\hline \multirow[b]{2}{*}{ Variables } & \multicolumn{3}{|c|}{ Univariable } & \multicolumn{3}{|c|}{ Multivariable } \\
\hline & $\mathbf{O R}$ & $95 \%$ CI & $P$ value* & OR & $95 \% \mathrm{CI}$ & $P$ value* \\
\hline Sex (male) & 2.00 & $1.08-3.74$ & .029 & 0.99 & $0.95-1.03$ & .667 \\
\hline Side (right) & 1.32 & $0.70-2.44$ & .399 & & & \\
\hline Location (peripheral) & 2.27 & $1.08-4.76$ & .032 & 1.61 & $0.69-3.85$ & .267 \\
\hline Lobe (upper) & 1.10 & $0.59-2.03$ & .772 & & & \\
\hline Pack-y of smoking & 0.98 & $0.97-0.99$ & .002 & 0.90 & $0.80-1.03$ & .072 \\
\hline COPD (stage I) & 2.06 & $0.89-4.76$ & .091 & & & \\
\hline CEA (ng/mL) & 0.86 & $0.78-0.96$ & .005 & 0.91 & $0.80-1.04$ & .112 \\
\hline SUVmax & 0.77 & $0.63-0.83$ & $<.001$ & 0.76 & $0.65-0.88$ & $<.001$ \\
\hline Maximum tumor size (mm) & 0.92 & $0.87-0.97$ & .002 & 1.01 & 0.94-1.09 & .763 \\
\hline Air bronchogram (absent) & 0.50 & $0.25-0.97$ & .041 & 0.53 & $0.25-1.14$ & .102 \\
\hline History of cancers (no) & 2.54 & $0.84-7.71$ & .100 & & & \\
\hline
\end{tabular}

The following factors were analyzed as a continuous variable: pack-y of smoking, carcinoembryonic antigen, SUVmax, and maximum tumor size. $O R$, Odds ratio; $C I$, confidence interval; $C O P D$, chronic obstructive pulmonary disease; $C E A$, carcinoembryonic antigen; $S U V m a x$, maximum standardized uptake value. $* P$ value in logistic regression analysis.

date of evaluating survival or recurrence was set as the end point of the RFS. Continuous variables that were normally distributed were summarized by mean and standard deviation; those not normally distributed were summarized by median and interquartile range.

\section{RESULTS}

The overall clinicopathologic characteristics of clinical stage IA radiologic pure-solid lung adenocarcinoma are summarized in Table 1. Histologic subtype of LPA was identified in approximately one third of the patients, the majority of which were lepidic predominant invasive adenocarcinomas, whereas the non-LPA subtypes were fairly evenly distributed.

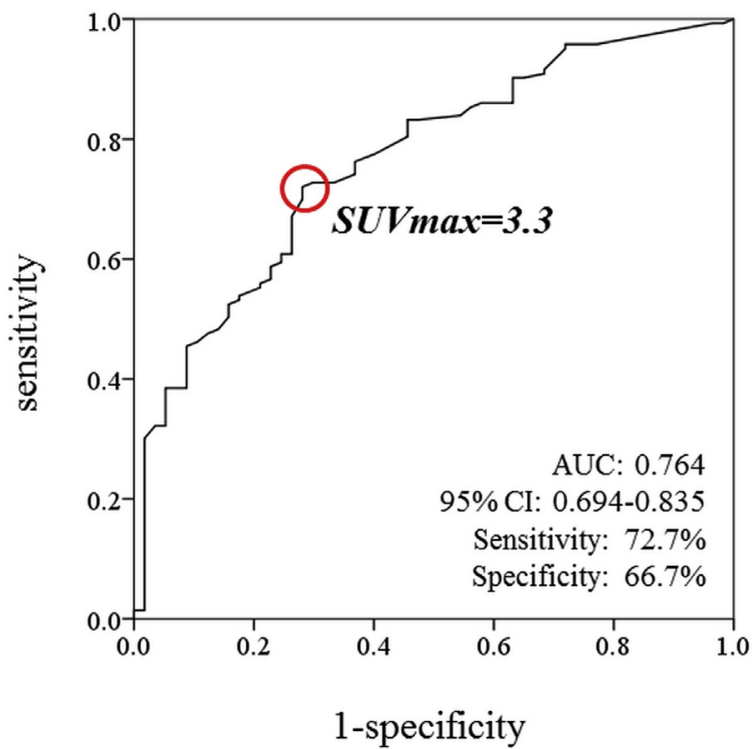

FIGURE 2. Receiver operating characteristic curve reveals that the optimal cutoff value for predicting LPA is an SUVmax of 3.3 (area under the curve, 0.764 ; sensitivity, $72.7 \%$; specificity, 66.7\%). AUC, Area under the curve; SUVmax, maximum standardized uptake value; $C I$, confidence interval.
Table 2 shows univariable and multivariable analyses for identifying lepidic predominant clinical stage IA pure-solid lung adenocarcinoma. From a multivariable analysis, SUVmax is an independent variable of LPA in this population $(P<.0001)$. The receiver operating characteristic curve revealed that the optimal cutoff value for identifying LPA was an SUVmax of 3.3 (Figure 2) (area under the curve, 0.764; sensitivity, $72.7 \%$; specificity, $66.7 \%$ ). Furthermore, the Cox proportional hazard analysis for the OS demonstrated that SUVmax $(P=.034)$ and pathologic nodal involvement $(P=.030)$ were independent significant prognostic factors (Table 3).

The 5-year OS and RFS of clinical stage IA radiologic pure-solid lung adenocarcinoma were $83.4 \%$ and $67.5 \%$, respectively, with a median follow-up period of 43.3 months for the OS and 41.8 months for the RFS. Furthermore, the 5year OS and RFS were significantly different between the LPA and non-LPA groups (5-year OS, $98.1 \%$ vs $76.6 \%$, $P=.0012$; 5-year RFS, $78.8 \%$ vs $59.9 \%, P=.0024$ ) (Figure E1). Furthermore, we divided clinical stage IA pure-solid lung adenocarcinomas into 2 categories based on SUVmax, that is, an SUVmax 3.3 or less arm $(n=77)$ and an SUVmax greater than $3.3 \mathrm{arm}(\mathrm{n}=123)$ based on the results of a multivariable analysis and receiver operating characteristic curve. Pathologically, lymphatic or vascular invasion, and nodal metastasis were less common in patients with an SUVmax 3.3 or less than those with an SUVmax greater than 3.3. LPA was shown in 38 patients $(49 \%)$ in the SUVmax 3.3 or less arm, but in 19 patients $(15 \%)$ in the SUVmax greater than 3.3 arm $(P<.0001)$. The 5 -year OS of the SUVmax 3.3 or less arm was significantly better than that of the SUVmax greater than 3.3 arm $(91.7 \%$ vs $78.6 \%, P=.0031)$. Furthermore, we investigated the clinicopathologic features in the 2 study arms based on the operative modes (Table 4). With regard to the oncologic outcomes, the 3-year locoregional RFS was similar between the sublobar resection arm $(\mathrm{n}=18)$ and the lobectomy arm 
TABLE 3. Cox proportional hazard analysis for overall survival

\begin{tabular}{|c|c|c|c|c|c|c|}
\hline \multirow[b]{2}{*}{ Variables } & \multicolumn{3}{|c|}{ Univariable } & \multicolumn{3}{|c|}{ Multivariable } \\
\hline & HR & $\mathbf{9 5} \% \mathbf{C I}$ & $P$ value* & HR & $\mathbf{9 5} \% \mathbf{C I}$ & $P$ value* \\
\hline Sex (male) & 0.59 & $0.26-1.35$ & .211 & & & \\
\hline Side (right) & 0.82 & $0.38-1.75$ & .604 & & & \\
\hline Location (peripheral) & 0.99 & $0.43-2.27$ & .991 & & & \\
\hline Lobe (upper) & 1.89 & $0.88-4.08$ & .103 & & & \\
\hline Pack-y of smoking & 1.01 & $0.99-1.01$ & .090 & & & \\
\hline COPD (stage I) & 0.59 & $0.26-1.36$ & .216 & & & \\
\hline CEA (ng/mL) & 1.04 & $1.02-1.06$ & $<.001$ & 1.02 & $0.99-1.04$ & .163 \\
\hline $\operatorname{SUVmax}(\leq 3.3)$ & 0.23 & $0.08-0.67$ & .007 & 0.27 & $0.08-0.91$ & .034 \\
\hline Maximum tumor size $(\mathrm{mm})$ & 1.07 & $1.00-1.14$ & .044 & 1.02 & $0.94-1.11$ & .612 \\
\hline Air bronchogram (absent) & 1.02 & $0.47-2.22$ & .966 & & & \\
\hline History of cancers (no) & 0.47 & $0.19-1.17$ & .107 & & & \\
\hline Operation (lobectomy) & 1.65 & $0.50-5.48$ & .416 & & & \\
\hline Nodal involvement (present) & 3.02 & $1.41-6.49$ & .004 & 4.24 & $1.15-15.6$ & .030 \\
\hline Pathologic stage (IA) & 0.525 & $0.24-1.13$ & .100 & & & \\
\hline Lymphatic invasion (present) & 3.18 & $1.39-7.30$ & .006 & 2.05 & $0.63-6.76$ & .236 \\
\hline Vascular invasion (present) & 2.70 & $1.18-6.17$ & .019 & 1.02 & $0.34-3.67$ & .965 \\
\hline LPA (no) & 12.86 & $1.74-94.9$ & .012 & 6.32 & $0.80-50.0$ & .081 \\
\hline
\end{tabular}

( $\mathrm{n}=59$ ) of patients with an SUVmax of 3.3 or less (Figure 3, A, 92.9\% vs 98.2\%, $P=.9761$ ), whereas that of the sublobar resection arm $(\mathrm{n}=17)$ was significantly worse than that of the lobectomy arm $(n=106)$ of patients with an SUVmax greater than 3.3 (Figure 3, B, 62.7\% vs $82.9 \%, P=.0281)$.

\section{DISCUSSION}

Our first study aim was to determine whether clinical factors can be used to identify lepidic growth in patients with clinical stage IA radiologic pure-solid lung adenocarcinomas. In general, radiologic pure-solid lung adenocarcinomas without a GGO component have been found to exhibit a more malignant behavior and have a poorer prognosis compared with part-solid lung adenocarcinomas. $^{4,9,10,19,20}$ However, the most critical finding in this study was that the SUVmax level correlated well with the histologic subtypes based on the IASLC/ATS/ETS classification even for cases of clinical stage IA radiologic pure-solid lung adenocarcinoma without GGO components

TABLE 4. Clinicopathologic characteristics based on the operative modes in each maximum standardized uptake value group

\begin{tabular}{|c|c|c|c|c|c|c|}
\hline \multirow{2}{*}{$\begin{array}{c}\text { Clinical factors } \\
\text { Operations } \\
\end{array}$} & \multicolumn{2}{|c|}{ SUVmax $\leq 3.3(N=77)$} & \multirow[b]{2}{*}{$P$ value $*$} & \multicolumn{2}{|c|}{ SUVmax $>3.3(N=123)$} & \multirow[b]{2}{*}{$P$ value } \\
\hline & Lobectomy $(n=59)$ & Sublobar $(n=18)$ & & Lobectomy $(n=106)$ & Sublobar $(\mathbf{n}=17)$ & \\
\hline Age (y) & $67.2 \pm 9.8(48-86)$ & $64.6 \pm 11.2(43-88)$ & .350 & $66.6 \pm 10.1(35-89)$ & $70.1 \pm 8.7(59-83)$ & .176 \\
\hline Sex (male) & $35(59)$ & $7(39)$ & .177 & $65(61)$ & $12(71)$ & .593 \\
\hline Location (peripheral) & $43(73)$ & $15(83)$ & .535 & $66(62)$ & $15(88)$ & .052 \\
\hline Pack-y smoking & $24.4 \pm 30.1(0-120)$ & $10.3 \pm 16.1(0-50)$ & .061 & $31.7 \pm 39.3(0-180)$ & $33.3 \pm 26.4(0-92)$ & .870 \\
\hline FEV $1 \%$ & $74.6 \pm 8.8(43-89)$ & $75.7 \pm 6.4(62-86)$ & .619 & $75.4 \pm 9.5(48-97)$ & $74.6 \pm 12.8(50-97)$ & .764 \\
\hline $\mathrm{VC}(\%)$ & $97.6 \pm 15.4(67-129)$ & $97.9 \pm 19.0(55-126)$ & .951 & $98.4 \pm 15.7(49-133)$ & $88.2 \pm 20.8(51-118)$ & .068 \\
\hline CEA (ng/mL) & $3.7 \pm 3.3(0.6-18.2)$ & $2.6 \pm 2.2(0.5-8.9)$ & .190 & $8.5 \pm 13.0(0.5-82.7)$ & $12.0 \pm 14.4(1.1-45.6)$ & .318 \\
\hline SUVmax & $2.2 \pm 0.8(0.7-3.3)$ & $1.5 \pm 0.7(0.7-3.2)$ & .0002 & $7.6 \pm 3.7(3.4-23.4)$ & $7.3 \pm 4.1(3.8-19.0)$ & .698 \\
\hline Maximum tumor size $(\mathrm{mm})$ & $19.0 \pm 8.0(7-30)$ & $10.012 .0(8-20)$ & $<.001$ & $21.4 \pm 5.6(10-30)$ & $18.8 \pm 25.4(12-28)$ & .073 \\
\hline c-T status (T1a) & $35(59)$ & $18(100)$ & $<.001$ & $50(47)$ & $12(71)$ & .111 \\
\hline Lymphatic invasion (present) & $20(34)$ & $2(11)$ & .077 & $58(55)$ & $6(35)$ & .191 \\
\hline Vascular invasion (present) & $19(32)$ & $2(11)$ & .129 & $70(66)$ & $3(18)$ & $<.001$ \\
\hline Nodal metastases (present) & $8(14)$ & $1(6)$ & .676 & $39(37)$ & $2(12)$ & .053 \\
\hline p-stage IA disease & $40(68)$ & $15(83)$ & .247 & $43(41)$ & $10(59)$ & .192 \\
\hline LPA & $26(44)$ & $12(67)$ & .112 & $16(15)$ & $3(18)$ & .726 \\
\hline
\end{tabular}

Categoric data are shown as numbers ( $\%)$, and continuous data are shown as mean \pm standard deviation if normally distributed and median \pm interquartile range if not normally distributed (range). The following factors were analyzed as a continuous variable: pack-y of smoking, FEV1\%, vital capacity (\%), CEA, SUVmax, and tumor size. SUVmax, Maximum standardized uptake value; $F E V 1$, forced expiratory volume in 1 second; $V C$, vital capacity; $C E A$, carcinoembryonic antigen; $L P A$, lepidic predominant adenocarcinoma. ${ }^{*} P$ value in chi-square test, Student $t$ test, or Wilcoxon rank-sum test. 
SUVmax $\leq 3.3$

$S U V \max >3.3$

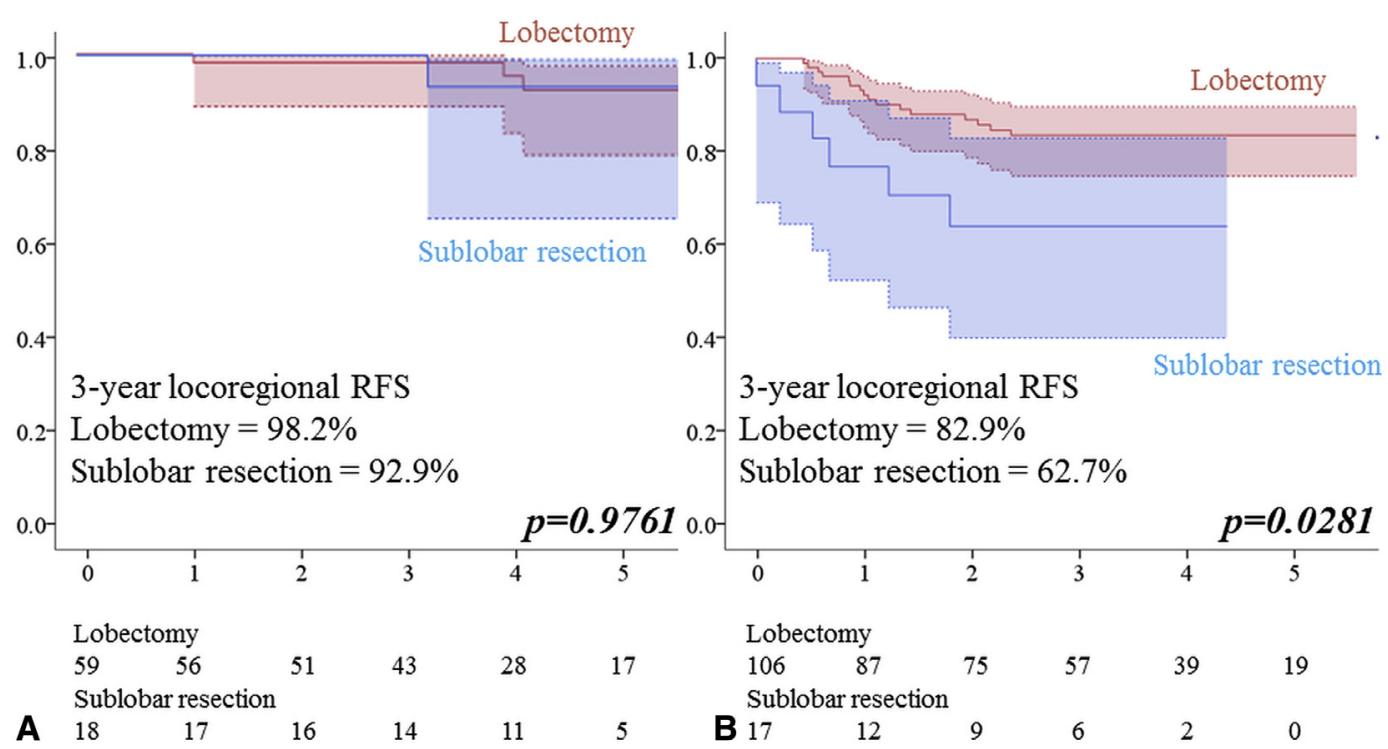

FIGURE 3. The 3-year locoregional RFS is presented on the basis of the operative mode. It is similar between the sublobar resection arm and the lobectomy arm of patients with an SUVmax of 3.3 or less (A, 92.9\% vs $98.2 \%, P=.9761)$, whereas that of the sublobar resection arm is significantly worse than that of the lobectomy arm of patients with an SUVmax of 3.3 or greater (B, $62.7 \%$ vs $82.9 \%, P=.0281)$. SUVmax, Maximum standardized uptake value; RFS, recurrence-free survival.

on thin-section CT scan, that is, SUVmax value was a promising clinical variable for identifying lepidic predominance among them.

These findings suggest that there are a subset of patients with adenocarcinoma who may be adequately treated with sublobar resection on the basis of clinical factors. To further explore this question, we stratified the patients on the basis of the SUVmax, which we identified as strongly associated with lepidic growth, to determine whether the approach to resection was associated with differential survival. Our finding that patients with a high SUVmax who underwent sublobar resection had worse survival suggests that higher SUVmax value may be useful for identifying patients with stage IA radiologic pure-solid lung adenocarcinoma in whom sublobar resection should not be considered.

Controversy still surrounds the appropriate operative modes for early-stage NSCLC with solid nodules. ${ }^{12,15,21-23}$ We speculate that the dispute regarding segmentectomy versus lobectomy for small-sized lung cancer to date would be due to a migration of part-solid and pure-solid tumors in the initial patient selection. The oncologic outcomes of the radiologic part-solid lung adenocarcinomas were excellent and definitely different from those of pure-solid tumors. ${ }^{4,24}$ Therefore, we focused exclusively on radiologic pure-solid tumors to address this matter. Moreover, pure-solid lung adenocarcinomas showed the histologic predominance of a wide variety of components including not only lepidic but also acinar, papillary, and solid as shown in the current study. Among them, lung adenocarcinomas demonstrating lepidic component predominance showed less invasiveness, and the oncologic outcomes of such adenocarcinomas are fully acceptable despite the operative mode used even for tumors that showed a radiologic pure-solid appearance.

Because of the refined resolution of thin-section CT scan, we can predict tumor invasiveness more precisely on the basis of the preoperative radiologic characteristics, namely, the presence of a GGO component. In routine practice, however, it is sometimes difficult to distinguish them on the basis of the findings on thin-section CT scan if the tumors have a radiologic pure-solid appearance because of the complete absence of a GGO component. In general, a radiologic puresolid appearance is considered to exhibit histologic nonlepidic components. However, there exists LPA showing a radiologic pure-solid appearance on thin-section CT scan because of the collapse of alveolar and intestinal fields. Thus, identifying LPAs is key to select patients with radiologic pure-solid lung adenocarcinoma for whom sublobar resection may be indicated.

Although definitive preoperative patient selection is mandatory especially when indicating sublobar resection for a radiologic pure-solid lung adenocarcinoma, our results showed that a lower SUVmax level of the tumor was a significant clinical factor of LPA, and patients who met our criterion showed less pathologic invasiveness and better OS than those who did not. Moreover, the 3-year locoregional RFS of the sublobar resection arm was significantly different than that of the lobectomy arm based on the SUVmax level of the tumor. This indicates that the SUVmax of 
tumors is a promising clinical parameter that may be useful for identifying patients in whom sublobar resection should not considered if the patient is a candidate for lobectomy, even if technically feasible, but this requires further study. The greatest concern regarding the indication for sublobar resection in patients with radiologic pure-solid lung adenocarcinoma is the potential risk for pathologic nodal metastasis and subsequent locoregional recurrence. Moreover, their frequencies are extremely high compared with those of part-solid tumors. Therefore, the decision to proceed with sublobar resection should be made with caution in patients with pure-solid lung adenocarcinomas.

SUVmax on PET and GGO appearances are significant preoperative variables for surgical outcome in clinical stage IA lung adenocarcinoma. ${ }^{8}$ Furthermore, we elucidated that SUVmax would be useful in quantitative assessments to subdivide radiologic pure-solid lung adenocarcinomas into lepidic predominant or nonlepidic predominant lesions in the current study. In principal, lobectomy with nodal dissection is the mainstay definitive locoregional surgical management for patients with clinical stage IA pure-solid lung adenocarcinomas, especially when it showed a high SUVmax (ie, SUVmax >3.3) because of its truly highly invasive nature. In contrast, segmentectomy may be an optimal surgical mode for cases with a low SUVmax because they have similar locoregional RFSs compared with cases of the lobectomy arm, although further validation study is necessary to confirm the result. Our primary objective was to explore the clinical factors correlated with the histologic lepidic growth component in this cohort, with the understanding that being able to determine the clinical variables that identify a high frequency of lepidic component would potentially validate the concept of selecting sublobar resection for clinical stage IA radiologic puresolid lung adenocarcinoma. On the basis of the results of the current study, SUVmax level of clinical stage IA radiologic pure-solid lung adenocarcinoma may provide a clue for identifying sublobar resection candidates in the future.

\section{Study Limitations}

This study was limited by its retrospective nature in a single center and the relatively small number of the patients undergoing sublobar resection for clinical stage IA radiologic pure-solid lung adenocarcinoma to prevent locoregional recurrence. In Japan, the prospective JCOG0802 study has completed enrolling cases, and the final result is awaited regarding appropriate operative modes for patients with radiologic invasive lung cancers. We hope to validate our recent results as an additional study in this prospective setting. Furthermore, variables of SUVmax on PET limit the generalizability of our results in daily practice. There are variations in SUVmax quantification among several institutions, and SUVmax is easily influenced by many factors, such as patient preparation procedures, scan acquisition, image reconstruction, and data analysis. To overcome this disadvantage, the standardized uptake value needs to be revised and PET procedures standardized. Thus, further investigations are warranted in the future.

\section{CONCLUSIONS}

Although any final decision regarding the efficacy of sublobar resection for radiologically invasive small-sized lung cancers should be based on the results of the prospective studies conducted by the JCOG and Cancer and Leukemia Group B, a lower SUVmax of the tumor was a significant clinical factor of histologically LPAs, and higher SUVmax value may be useful for identifying patients with clinical stage IA radiologic pure-solid lung adenocarcinoma in whom sublobar resection should not be considered, even if it is technically feasible.

\section{Conflict of Interest Statement}

Authors have nothing to disclose with regard to commercial support.

\section{References}

1. Siegel R, Ma J, Zou Z, Jemal A. Cancer statistics, 2014. CA Cancer J Clin. 2014; 64:9-29.

2. Travis WD, Brambilla E, Noguchi M, Nicholson AG, Geisinger KR, Yatabe Y, et al. International Association for the Study of Lung Cancer/American Thoracic Society/European Respiratory Society International Multidisciplinary Classification of Lung Adenocarcinoma. J Thorac Oncol. 2011;6:244-85.

3. Aokage K, Yoshida J, Ishii G, Matsumura Y, Haruki T, Hishida T, et al. Identification of early $\mathrm{t} 1 \mathrm{~b}$ lung adenocarcinoma based on thin-section computed tomography findings. J Thorac Oncol. 2013;8:1289-94.

4. Hattori A, Matsunaga T, Takamochi K, Oh S, Suzuki K. Neither maximum tumor size nor solid component size is prognostic in part-solid lung cancer: the impact of tumor size should be applied exclusively to solid lung cancer. Ann Thorac Surg. 2016;102:407-15.

5. Saji H, Matsubayashi J, Akata S, Shimada Y, Kato Y, Kudo Y, et al. Correlation between whole tumor size and solid component size on high-resolution computed tomography in the prediction of the degree of pathologic malignancy and the prognostic outcome in primary lung adenocarcinoma. Acta Radiol. 2015;56: $1187-95$.

6. Suzuki K, Asamura H, Kusumoto M, Kondo H, Tsuchiya R. "Early" peripheral lung cancer: prognostic significance of ground glass opacity on thin-section computed tomographic scan. Ann Thorac Surg. 2002;74:1635-9.

7. Suzuki K, Koike T, Asakawa T, Kusumoto M, Asamura H, Nagai K, et al. A prospective radiological study of thin-section computed tomography to predict pathological noninvasiveness in peripheral clinical IA lung cancer (Japan Clinical Oncology Group 0201). J Thorac Oncol. 2011;6:751-6.

8. Okada M, Nakayama H, Okumura S, Daisaki H, Adachi S, Yoshimura M, et al Multicenter analysis of high-resolution computed tomography and positron emission tomography/computed tomography findings to choose therapeutic strategies for clinical stage IA lung adenocarcinoma. J Thorac Cardiovasc Surg. 2011;141:1384-91.

9. Hattori A, Matsunaga T, Takamochi K, Oh S, Suzuki K. Oncological character istics of radiological invasive adenocarcinoma with additional ground-glass nodules on initial thin-section computed tomography: comparison with solitary invasive adenocarcinoma. J Thorac Oncol. 2016;11:729-36.

10. Hattori A, Suzuki K, Matsunaga T, Fukui M, Kitamura Y, Miyasaka Y, et al. Is limited resection appropriate for radiologically "solid" tumors in small lung cancers? Ann Thorac Surg. 2012;94:212-5.

11. Suzuki K, Kusumoto M, Watanabe S, Tsuchiya R, Asamura H. Radiologic classification of small adenocarcinoma of the lung: radiologic-pathologic correlation and its prognostic impact. Ann Thorac Surg. 2006;81:413-9. 
12. Altorki NK, Yip R, Hanaoka T, Bauer T, Aye R, Kohman L, et al. Sublobar resection is equivalent to lobectomy for clinical stage $1 \mathrm{~A}$ lung cancer in solid nodules. J Thorac Cardiovasc Surg. 2014;147:754-64.

13. Landreneau RJ, Normolle DP, Christie NA, Awais O, Wizorek JJ, Abbas G, et al. Recurrence and survival outcomes after anatomic segmentectomy versus lobectomy for clinical stage I non-small-cell lung cancer: a propensity-matched analysis. J Clin Oncol. 2014;32:2449-55.

14. Okada M, Koike T, Higashiyama M, Yamato Y, Kodama K, Tsubota N. Radical sublobar resection for small-sized non-small cell lung cancer: a multicenter study. J Thorac Cardiovasc Surg. 2006;132:769-75.

15. Tsutani Y, Miyata Y, Nakayama H, Okumura S, Adachi S, Yoshimura M, et al. Oncologic outcomes of segmentectomy compared with lobectomy for clinical stage IA lung adenocarcinoma: propensity score-matched analysis in a multicenter study. J Thorac Cardiovasc Surg. 2013;146:358-64.

16. Nakamura K, Saji H, Nakajima R, Okada M, Asamura H, Shibata T, et al. A phase III randomized trial of lobectomy versus limited resection for small-sized peripheral non-small cell lung cancer (JCOG0802/WJOG4607L). Jpn J Clin Oncol. 2011;40:271-4.

17. ClinicalTrials.gov. Bethesda, MD: National Institutes of Health; Comparison of different types of surgery in treating patients with stage IA non-small cell lung cancer. Available at: http://clinicaltrials.gov/ct/show/NCT00499330. Accessed January 9, 2012.

18. Goldstraw P, Crowley J, Chansky K, Giroux DJ, Groome PA, Rami-Porta R, et al. The IASLC Lung Cancer Staging Project: proposals for the revision of the TNM stage groupings in the forthcoming (seventh) edition of the TNM Classification of malignant tumours. J Thorac Oncol. 2007;2:706-14.
19. Hattori A, Suzuki K, Matsunaga T, Miyasaka Y, Takamochi K, Oh S What is the appropriate operative strategy for radiologically solid tumours in subcentimetre lung cancer patients? Eur J Cardiothorac Surg. 2015;47: 244-9.

20. Hattori A, Suzuki K, Matsunaga T, Takamochi K, Oh S. Visceral pleural invasion is not a significant prognostic factor in patients with a part-solid lung cancer. Ann Thorac Surg. 2014;98:433-8.

21. Khullar OV, Liu Y, Gillespie T, Higgins KA, Ramalingam S, Lipscomb J, et al Survival after sublobar resection versus lobectomy for clinical stage IA lung cancer: an analysis from the National Cancer Data Base. J Thorac Oncol. 2015;10: 1625-33.

22. Tsutani Y, Miyata Y, Nakayama H, Okumura S, Adachi S, Yoshimura M, et al. Segmentectomy for clinical stage IA lung adenocarcinoma showing solid dominance on radiology. Eur J Cardiothorac Surg. 2014; 46:637-42.

23. Kodama K, Higashiyama M, Okami J, Tokunaga T, Imamura F, Nakayama T, et al. Oncologic outcomes of segmentectomy versus lobectomy for clinical T1a N0 M0 non-small cell lung cancer. Ann Thorac Surg. 2016; 101:504-11.

24. Hattori A, Matsunaga T, Takamochi K, Oh S, Suzuki K. Locoregional recurrence after segmentectomy for clinical-T1aNOM0 radiologically solid non-small cell lung carcinoma. Eur J Cardiothorac Surg. 2017;51:518-25.

Key Words: lobectomy, sublobar resection, pure-solid tumor, lung adenocarcinoma, prognosis 